\title{
OPTICAL MEMORY
}

\section{The secret of the stone}

While casually sipping his

Martini, a secret agent scans the glamorous guests filling the room. His eyes rest on an innocent-looking woman in a simple dress adorned with a single pale stone. Has he recognized that the stone is the key to all the information that is holding his adversary's evil organization together?

Yuko Ishii et al. would have recognized this hidden device. They have developed a holographic information storage system in which a piece of the mineral ulexite is a key component ( $Y$. Ishii et al. Opt. Express 15, 7218-7223; 2007). As each stone is unique and irreproducible, only the stone that is used to store the information can retrieve it again.

In holographic memory, information is stored optically in a light-sensitive crystal. An 'object' laser beam (containing information) produces an interference pattern with a reference beam, and the pattern is inscribed on the crystal. Information is retrieved again by using a third beam, in combination with the reference laser.

The beauty of the technique is that multiple images can be stored in the same volume, by slightly changing the settings of the reference beam at each recording step.

Although it cannot yet beat magnetic disks in terms of price and performance, holographic data storage has its own advantages, and could become more competitive if its storage capacity could be improved. One way to achieve this is to use random reference beams, which can be generated by randomly rotating a diffuser in front of the reference laser. But this requires
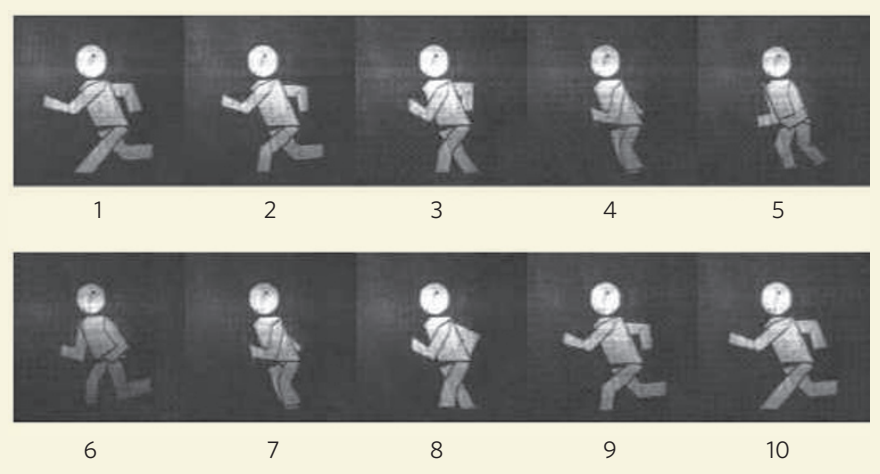

a computer-controlled rotator and an algorithm to impose the randomness.

Ishii and colleagues' approach is much simpler and more compact. They use a piece of ulexite as the diffuser and rotate it in regular steps. Because each piece of mineral has a unique composition, the random pattern of reference beams produced cannot be replicated by any other stone. Furthermore, only that stone can be used to retrieve the data. Ishii et al. demonstrate the effectiveness of the scheme by holographically storing images of a running figure and retrieving them as an animation with six frames per second (see figure).

The authors note that the stone could be worn as part of an accessory and so be carried without attracting suspicion except, that is, from a secret agent who has kept up with the scientific literature.

Liesbeth Venema occurred within two months to provide the quality of preservation seen in the mine.

Volcanic ash falls and mud flows are another, and demonstrably instant, method by which vegetation can be buried and preserved. Indeed, many fossil plant sites characterized by superb preservation seem to have a component of volcanic ash deposition in their genesis. For example, Wing et al. ${ }^{2}$ described a 4-kilometre-long transect through a Late Cretaceous meadow, dating to about 71.7 million years ago, that had been preserved in a 2-4-metre-thick slurry of volcanic ash. In this case, not only arboreal but also herbaceous vegetation was found rooted where it grew. Wing et al. were able to map the vegetation according to soil type, and to show that angiosperm herbs were more prevalent in better drained sites, whereas ferns and cycads trended towards the mires.

These 'plant Pompeiis' have an additional advantage in that they are preserved in a medium that is itself amenable to radiometric dating. Volcanic ash beds often contain sanidine or zircon crystals that formed in magma chambers shortly before eruption, trapping radioactive potassium and uranium, respectively. Ash-bed floras are literally buried in a matrix of fossil 'clocks'. As the precision of dating techniques increases, the ability to resolve the age of in situ deposits also increases. Because of better mass spectrometers and laboratory practices, error bars have been getting smaller during the past few years, and errors at the two-sigma statistical level have crept down from $0.5 \%$ to $0.1 \%$, and in some labs are heading for $0.05 \%$ or even less ${ }^{3,4}$.

EARTHTIME $^{5,6}$ is an international project intended to consolidate these gains by reducing interlaboratory variability, and to understand the differences in precision and accuracy of different dating methods (especially between uranium-lead and argon-argon geochronology). This precision is being combined with the ability of the astronomical timescale to define individual 22,000-year Milankovitch cycles $^{7}$, which are due to variation in Earth's orbit. All in all, we can look forward to a time when events that occurred as long ago as the beginning of the Cretaceous, some 145 million years ago, can be directly and precisely measured in thousands of years rather than hundreds of thousands or millions of years. Sedimentological evidence for instantaneous deposition could then pin-point an event to a period of a few months or less within the window of a few thousand years.

DiMichele et al. ${ }^{1}$ have taken advantage of the near-instantaneous deposition, superb preservation and great extent of their fossil forest to statistically analyse the distribution of different tree species across an ancient landscape. Carboniferous rainforests were composed of completely different major tree groups from those that exist today. For example, lycopsids (Fig. 1), the largest trees in these forests, stood above understories of tree ferns, pteridosperms (extinct seed ferns), cordaitaleans (extinct seed plants related to conifers) and sphenopsids (trees related to living horsetail rushes). The authors' analysis shows that the ecology of these long-extinct forests can be retrieved, and that forest heterogeneity on the local scale can be resolved from ecological gradients on a landscape scale.

There is good evidence that vegetation is in dynamic equilibrium with climate ${ }^{8}$, and it is becoming clear that some climate change can occur on a subdecadal timescale ${ }^{9}$. In circumstances in which a fossil site has been buried over a period of between hours and months, with climate change occurring in decades and geochronological resolution improving by the day, we are entering a world in which the term 'geological time' is becoming less relevant and we are forging the tools to resolve rapid events in real time. A new partnership between palaeontologists and geochronologists will revolutionize the way we think about rates of change - even for events that occurred hundreds of millions of years ago. Just because something happened a long time ago does not mean it took a long time to happen.

Kirk R. Johnson is at the Denver Museum of Nature and Science, 2001 Colorado Boulevard,

Denver, Colorado 80205, USA.

e-mail: kirk.johnson@dmns.org

1. DiMichele, W. A., Falcon-Lang, H. J., Nelson, W. J., Elrick, S. D. \& Ames, P. R. Geology 35, 415-418 (2007).

2. Wing, S. L., Hickey, L. J. \& Swisher, C. C. Nature 363, 342-344 (1993).

3. Storey, M., Duncan, R. A. \& Swisher, C. C. Science 316, 587-589 (2007)

4. Furin, S. et al. Geology 34, 1009-1012 (2006).

5. Kerr, R. A. Science 302, 375 (2003).

6. www.earthtime.org

7. Kuiper, K. F., Wijbrans, J. R. \& Hilgen, F. J. Terra Nova 17, 385-398 (2005)

8. Webb, T. Vegetatio 67, 75-91 (1986).

9. Alley, R. B. Quat. Sci. Rev. 19, 213-226 (2000).

\section{Correction}

In the caption to Figure 1 of the News \& Views article "Planetary science: Cracks under stress" by Andrew J. Dombard (Nature 447, 276-277; 2007), the number for the conversion of 91 kelvin to centigrade should, of course, be $-182^{\circ} \mathrm{C}$. 